\title{
Smart grids: enabler for the energy transition
}

\author{
Diana Moneta
}

Ricerca sul Sistema Energetico-RSE SpA - Via Rubattino 54, 20134 Milano, Italy

\begin{abstract}
Summary. - This paper deals with the concept of "smart grids". Starting from drivers behind the evolution of the power system, an overview of elements and technologies is given: smart grids have to intelligently integrate the actions of all users connected to it - generators, consumers and those that do both - in order to efficiently deliver sustainable, economic and secure electricity supplies. Examples of solutions from demonstration projects represent the base for discussing methodologies to estimate benefits deriving from smart grid solutions, showing how conflicting goals cannot always be fulfilled simultaneously. Finally, future perspectives are discussed: the main thinking regards the awaited pivot role for customers, in the future scenario with an increased utilization of electricity combined with a high penetration of non-programmable resources.
\end{abstract}

\section{1. - Smart grid: drivers}

The European Union in 2007 has started a comprehensive plan to increase the environmental sustainability, the security of supply and the competitiveness of its energy system. This strategy was translated into binding targets for greenhouse gases reduction, renewable energy sources (RESs) and energy efficiency (the so called "20-20-20" package).

In most EU countries, these goals have required a major contribution from electrical final uses in order to compensate lower efforts achievable in transports and thermal 
usages. In Italy, for example, to reach a 17\% of gross final energy uses in 2020 covered by renewable resources, a $26.39 \%$ share of electric uses was expected to balance their smaller penetration in other sectors [1]. In fact, the nature of the electricity vector allows to integrate more easily RESs, even if electricity counts for less than one third of the total energy consumption in Europe. Analogous strategies are followed by extra EU countries, depending on the availability of resources and on the market.

In the past decade, the exploitation of renewable sources has been realized by means of small-size generators especially, with a leading role of photovoltaic plants and wind farms and a more limited growth of biomass and run-of-river (without basin) hydro plants. Medium and small generators connected to the distribution grid are referred to as "distributed generation"; some definitions include a power threshold but the typology of the hosting network represents the focus for the scope of this discussion.

The presence of a large number of plants connected to the distribution networks has caused the entire system to undergo a deep evolution, towards the so-called "smart grid" concept. According to the EU definition, a smart grid is a "grid that can intelligently integrate the actions of all users connected to it - generators, consumers and those that do both - in order to efficiently deliver sustainable, economic and secure electricity supplies" $\left({ }^{1}\right)$.

\section{2. - Elements and technologies}

The electric system is probably the largest machine built by humankind. It binds in a single, interconnected network the European countries with the neighbouring continent, supplying citizens and industries [2].

Electricity is an energy vector and it cannot be stored without converting it into another form (chemical, mechanical, etc.). Then, in every time instant, the balance between the energy absorption from loads and the energy injection by generators has to remain very accurate, and only small variations around the (dynamic) equilibrium point are admissible. Since the power system is based on the alternating current (AC) concept [2], the frequency is maintained around $50 \mathrm{~Hz}(60 \mathrm{~Hz}$ in USA and other countries). Analogously, the voltage must be kept within its allowed range [3] to have appliances and devices operating properly.

This system was designed decades ago in an "unidirectional" framework, where large bulk power plants, usually based on coal, hydro, nuclear or gas, inject the electricity into the high voltage (HV) transmission lines, then this energy is delivered to the medium and to the low voltage distribution grid to reach the final users. In fig. 1 a simplified scheme of the power system is given: Primary and secondary substations convert the energy to/from the different voltage levels: in transmission lines the voltage $V$ is higher in order to reduce the losses (inversely proportional to $V$ for a given power transfer) while loads in customers' premise operate at low voltage.

$\left.{ }^{1}\right)$ Firstly proposed by the Electricity Networks Strategy Group (ENSG), UK. 


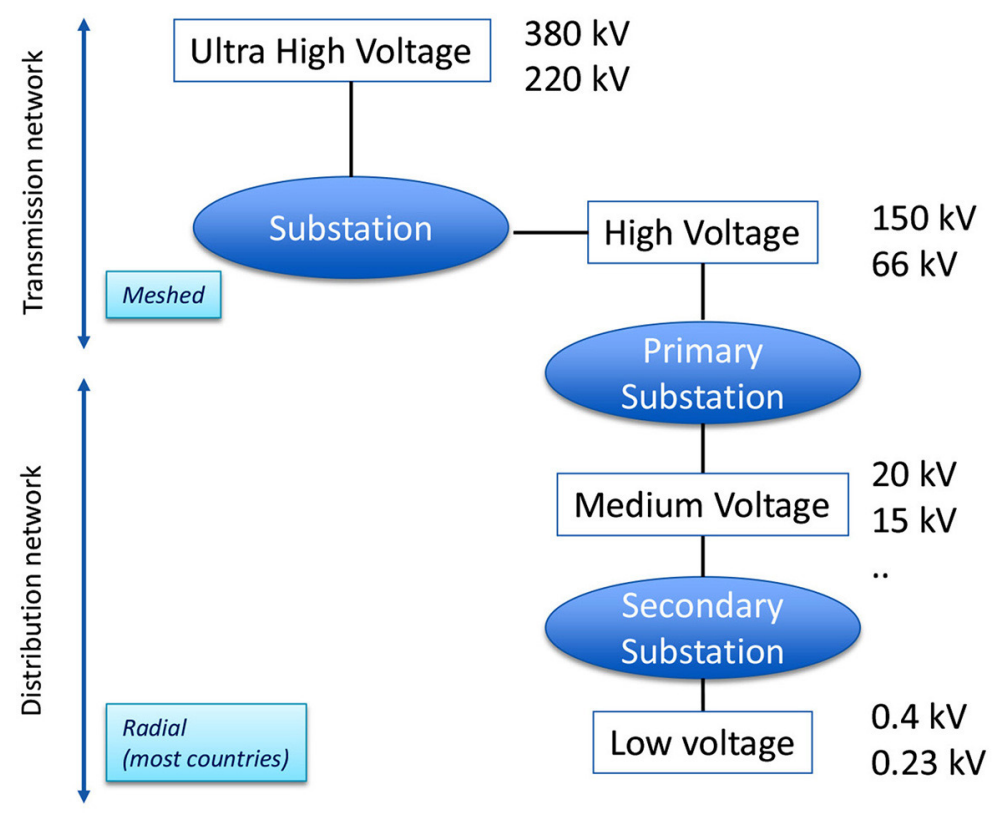

Fig. 1. - Simplified scheme of the power system (voltage levels may vary in the different countries).

The operation of the power system shows differences among the countries, the basic one is that the transmission networks are operated in a meshed topology while distribution grids are usually operated radially even if re-configurations are applied to face faults. Figure 2 schematizes the boundary between transmission and distribution grid, that is the primary substation (PS) where the power is converted from the High Voltage (HV) to the Medium Voltage (MV) level. Usually there are two transformers, working each at less than half of the nominal power to have a margin for outages or maintenance of the other one. Some transformers are equipped with the on load tap changer (OLTC) which allows varying the voltage of the MV busbar through the transformation ratio ("taps" are the connection points along the winding).

As mentioned before, most Distribution System Operators (DSO) manage their MV network radially even if the grid topology is meshed (fig. 3): in case of outages, automatic procedures exclude the fault section of the network and counterfeed the remaining parts through the working lines, reducing the impact on the customers. Besides, thanks to planning tools, the topology can be optimized to balance the load among the different feeders, for efficiency purposes also. With this architecture, only remote rural loads served by single lines may experience longer outages.

The secondary substations feed the MV customers and, through a transformer, the LV customers (fig. 4). Each secondary substation serves from few users (rural areas) to hundreds of premises (urban centres). The transformer characteristics are selected as a function of the total power of customers, considering the probability of simultaneous 


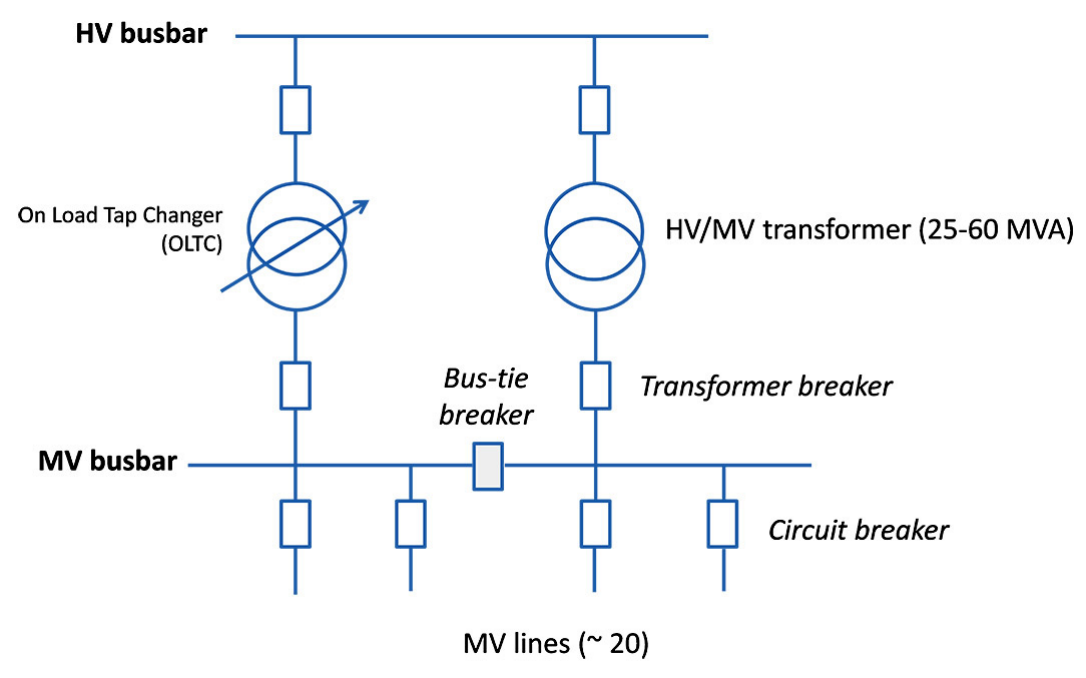

Fig. 2. - Simplified scheme of a HV/MV Primary Substation (PS). PSs usually have 1 to 3 transformers, and in some PSs transformers are equipped with OLTC (on load tap changer) to vary the voltage of the MV busbar independently of the HV busbar. The bus-tie breaker is usually open, it allows to connect all the MV lines under a single transformer in case of outages or maintenance of the second equipment.

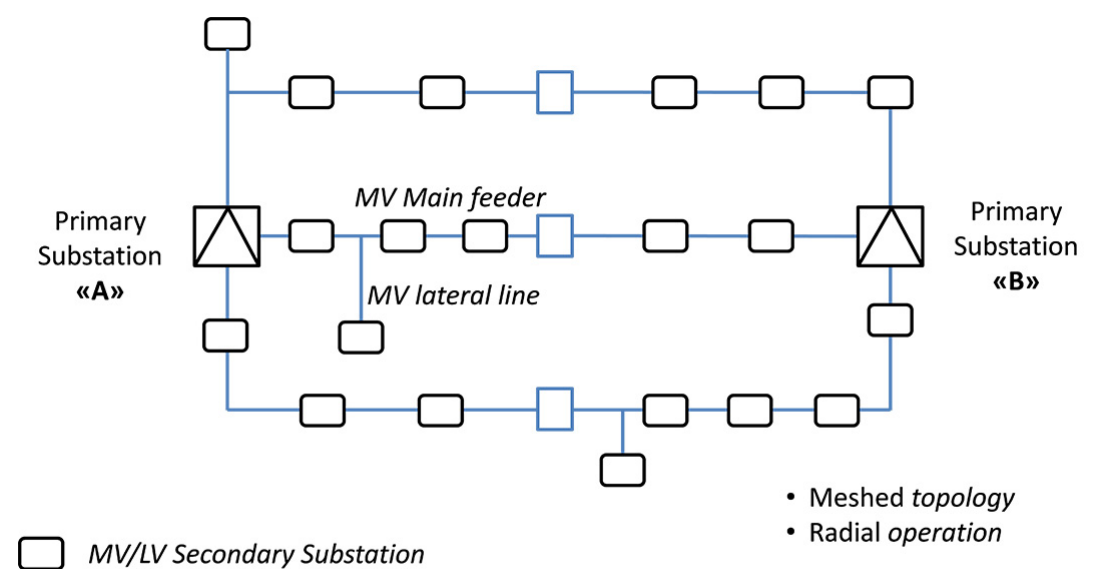

MV/LV Secondary Substation

- Radial operation

\section{Normally open points -Secondary Substation (remotely controlled)}

Fig. 3. - Simplified scheme of medium-voltage networks. In case of a fault, the normally open points are closed to re-configure the topology, counterfeeding MV/LV substations from the other Primary substation.

utilization of the loads. The architecture based on the two levels of substations is "scalable" to cover properly the different areas; on the average there is a 200:1 ratio between the number of secondary and primary substations. 


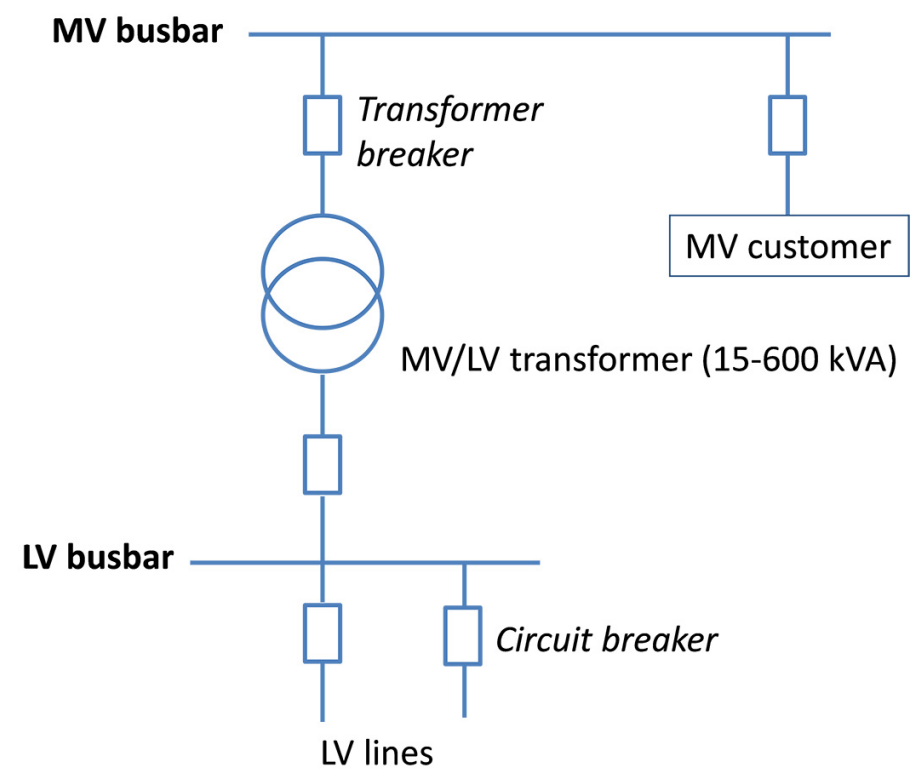

Fig. 4. - Simplified scheme of a MV/LV Secondary Substation (SS).

In very few words, the Transmission System Operator (TSO) is in charge of the security of supply and balancing, so it keeps the frequency around $50 \mathrm{~Hz}$ (in Europe, $60 \mathrm{~Hz}$ in USA and other countries) acting on balancing resources, acquired on the ancillary service market. Traditionally, this flexibility has been provided by bulk generators able to modulate their active power production within few seconds, in order to counterbalance the frequency deviations. In addition, thermal power plants have large rotating masses that pose a significant inertia to the variations of the power frequency.

To avoid duplication of the infrastructure, for a given geographical area there is only one DSO in charge of the supply of electricity. It has the obligation, deriving from the concession, of connecting all the customers, and it has to guarantee an adequate quality of supply in terms of continuity and stability of the voltage, and to keep currents in lines below their rated value. Besides, in most countries DSOs are in charge of metering, that is the collection of consumption data for billing purposes: these data are delivered to the vendor, or retailer, e.g. the commercial counterpart of customers for the electricity supply. According to EU rules, DSO and the vendor are separate entities so customers can choose the preferred contract on the market.

Automation has strongly contributed to improve the continuity and quality of supply in the past years, having a significant reduction of the number and the duration of outages. In fig. 5 the trend in the Italian case is reported: it can be seen how, thanks to the premium/penalty scheme set by the National Regulation Authority, the overall average duration of interruptions attributed to DSOs has been reduced; conversely, the interruptions deriving from other causes (weather, fraud) have increased in the recent years. Similar patterns are observable in other countries. 


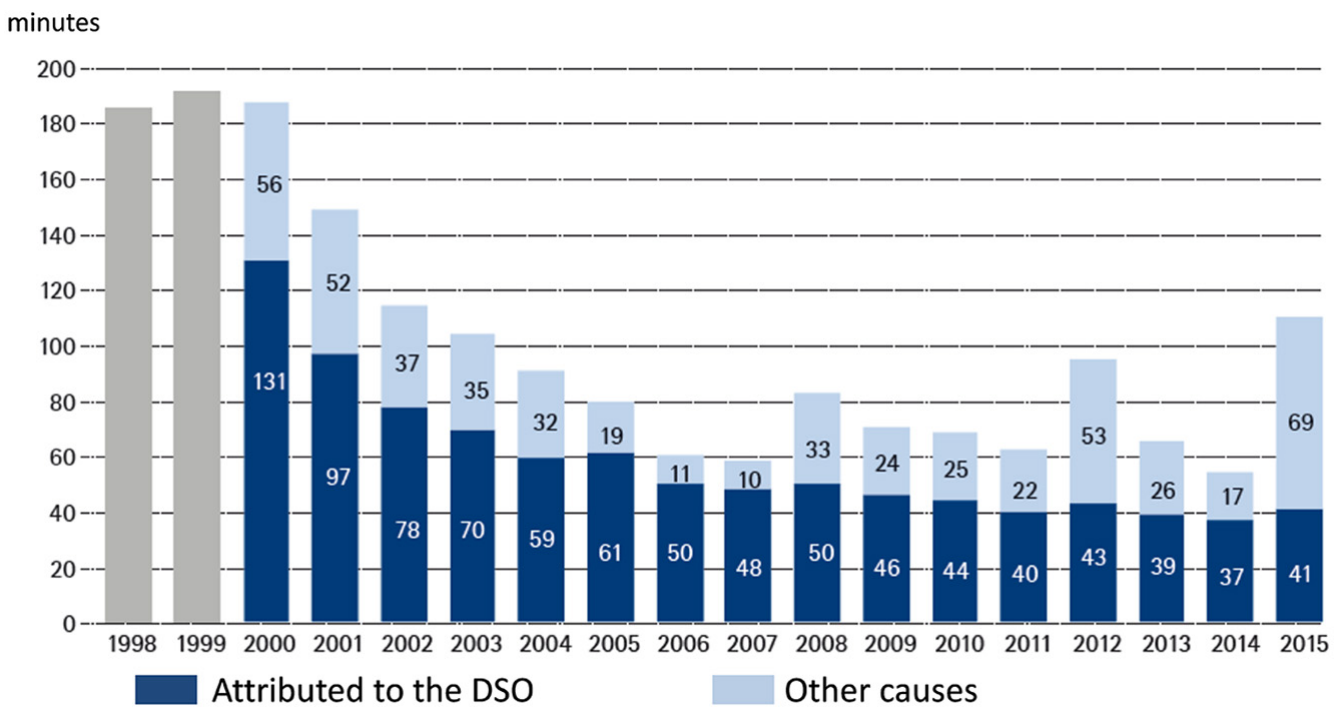

Fig. 5. - Long interruptions without notice - average duration per Low-Voltage Italian customer. Dark bars represent interruptions attributed to DSO and included into the penalty/premium scheme, other causes include weather events and frauds (source: Italian National Regulation Authority for Energy [4]).

As mentioned before, the support to RES-based generation, implemented through implicit and explicit incentives $\left({ }^{2}\right)$, has led to a high penetration of Distributed Generation (GD) in MV and LV networks.

Besides the environmental aspects, the presence of DG has other tangible effects: firstly, generation is installed close to loads, with less involvement of the transmission lines $\left({ }^{3}\right)$. In particular, PV production occurs in daylight hours (when the load is greater) and reaches a peak in the summer season (when the load is higher, as is the case in Italy). During the middle of the day the energy prices are lowered, due to the PV energy production: for the year 2014, the estimated price reductions on the Italian day ahead market was between 5.8 and $24 € / M W h$, with savings for consumers accounting to $896 \mathrm{M} €$. These effects on the prices is evident around $2 \mathrm{PM}$ in fig. 6, which plots the change in the day ahead market electricity prices over several years.

The impact of DG on the distribution system has been deeply discussed in the literature. Up to a few years ago, DGs were connected under the so-called "fit \& forget" approach, where no functional contribution from generators was expected towards the system. With large generation units, the transmission system operator is able to monitor

$\left({ }^{2}\right)$ See the contribution by Clerici to this School.

$\left({ }^{3}\right)$ It is worth mentioning that the power system was born in a decentralized architecture: at the beginning generators were built close to buildings and factories, and only when alternating current transmission technologies became available, power plants were located further from towns. 


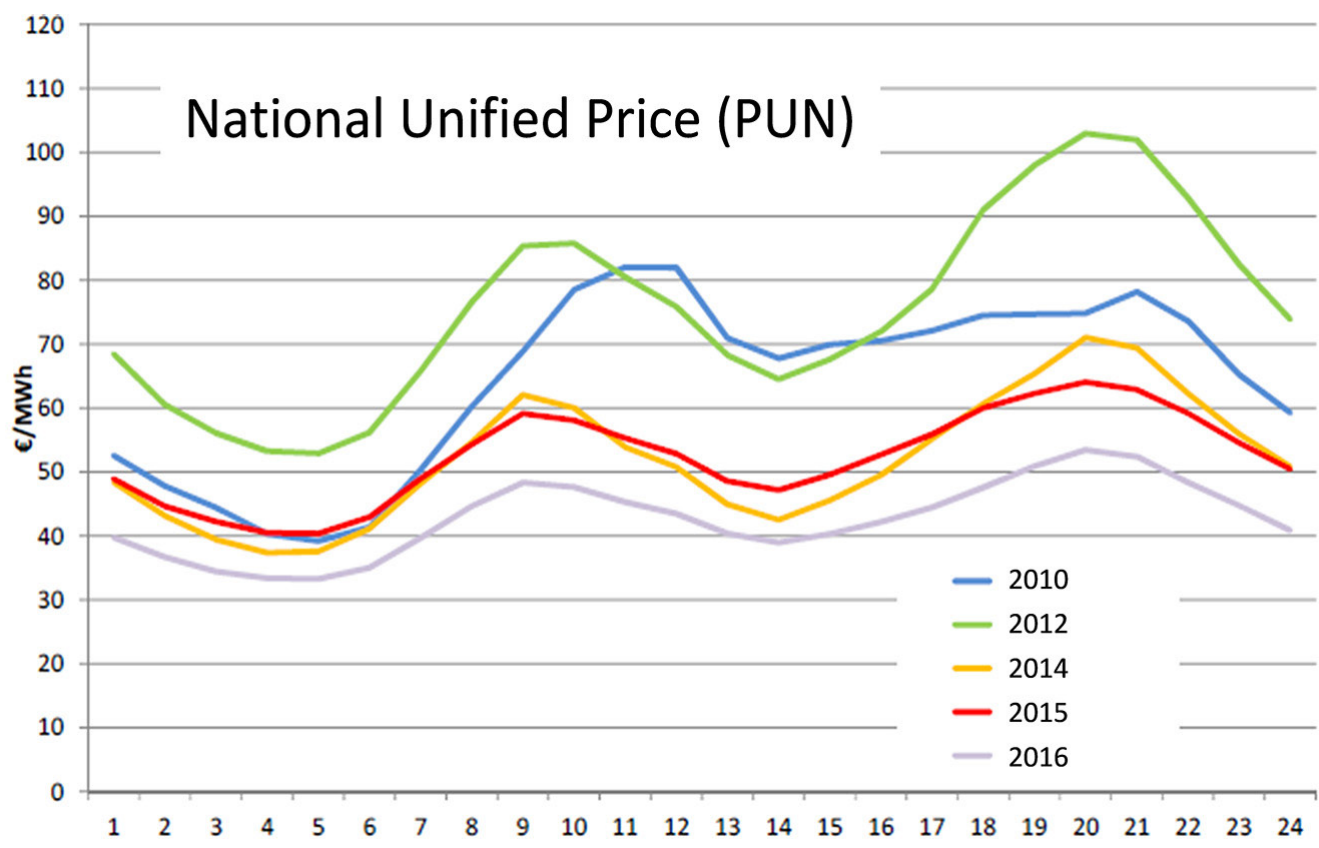

Fig. 6. - Effect of PV production on the day ahead market price in Italy, working days. In 2010 the total PV capacity was $3.47 \mathrm{GW}$ while in 2013 it had already exceed $18 \mathrm{GW}$ (elaboration from GME data).

and plan the production (ordering a curtailment if needed, producers still get paid as usual), while distributed generation, according to the traditional approach, cannot be dispatched in this way: network operators are legally forced to accept their production whatever it is. This may lead to limit the diffusion of generators, given the most valuable effects they could entail at both local and global levels:

- Voltage variations: injection of active power increases the voltage profile in the neighbouring nodes. Voltage rise represents actually the main limitation to the penetration of DG in rural areas [5].

- Harmonic distortion (noise at a multiple of the fundamental frequency $50 \mathrm{~Hz}$ ); this can interfere with power line communication used for of smart meters [6].

- Grid overload (thermal limits of transformers and lines).

- Impact on protection devices (interference with the automatic procedure of identification and exclusion of faulty areas).

- Poor observability of the system: generators are connected to networks not monitored by the TSO. This combines with the lack of programmability of most RES generation, entailing the availability of additional flexibility resources ("reserve") 


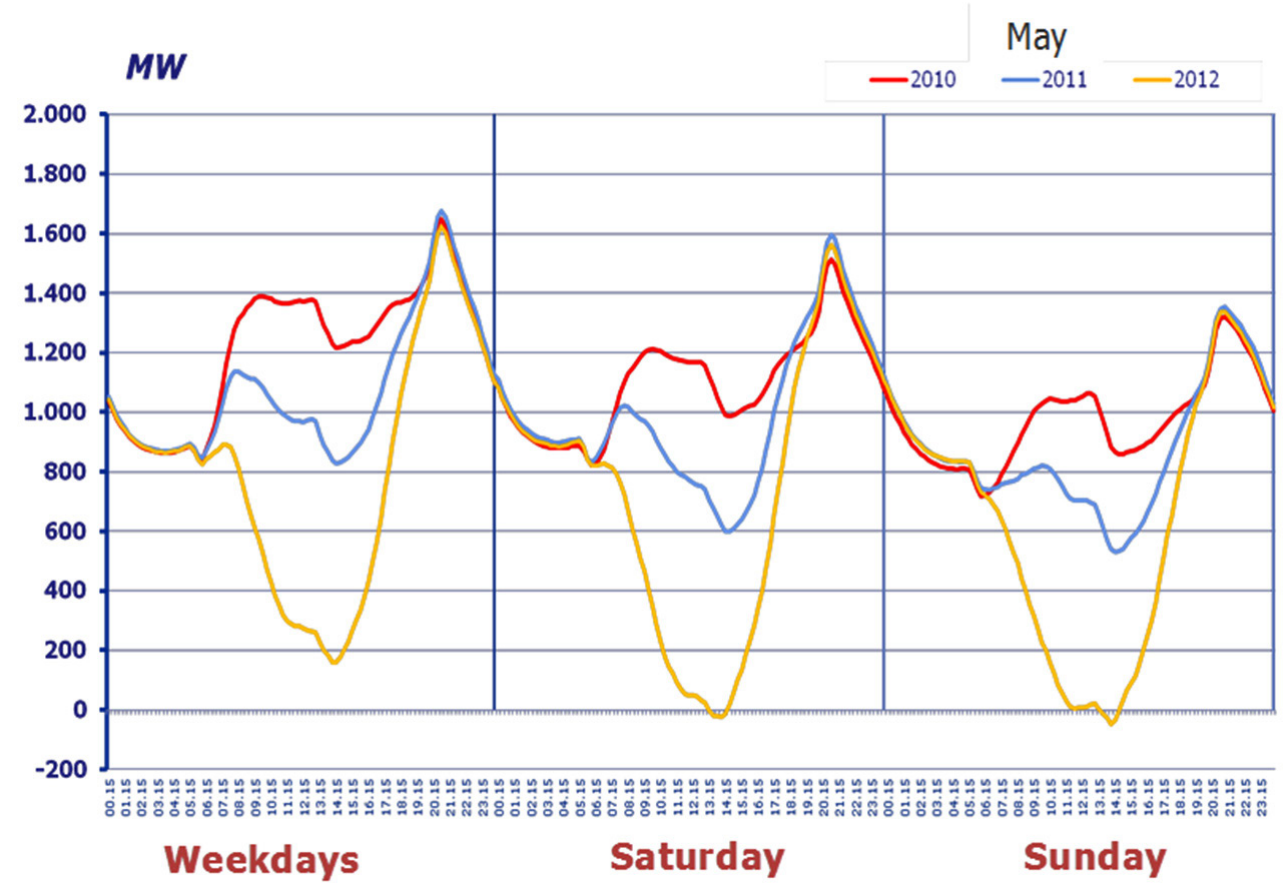

Fig. 7. - "Summer sunny day syndrome": in several HV/MV substations located in areas with high PV penetration, the power exchange from the transmission level to the distribution network has reduced and even reversed in the central hours. This graph refers to the Puglia region, in Southern Italy (source: ENEL). In 2016, more than $25 \%$ of primary substations had a reverse flow for at least $1 \%$ of hours.

to compensate real time fluctuations of primary sources. Bulk generators, the traditional provider of such flexibility, are progressively displaced from the market because of low prices $\left({ }^{4}\right)$ : some gas fired plants are kept online only for providing this balancing service.

- Reverse flow from the MV to the transmission networks (fig. 7). With high presence of $\mathrm{PV}$ production, the net load during the middle of the day is lowered, while in the afternoon it follows a steep rise because the reduction in solar radiation combines with the evening load peak (Summer syndrome, fig. 7).

- Reduced inertia. RES based generators, connected to the system with power electronic interfaces, displace traditional thermal plants so variations of the frequency are not effectively dumped. Besides, before updating the connection rules, DGs could be disconnected during over - and under — frequency events, worsening the unbalancing and contributing to large blackouts in Europe [7].

$\left({ }^{4}\right)$ See the contribution by Erdmann to this School. 

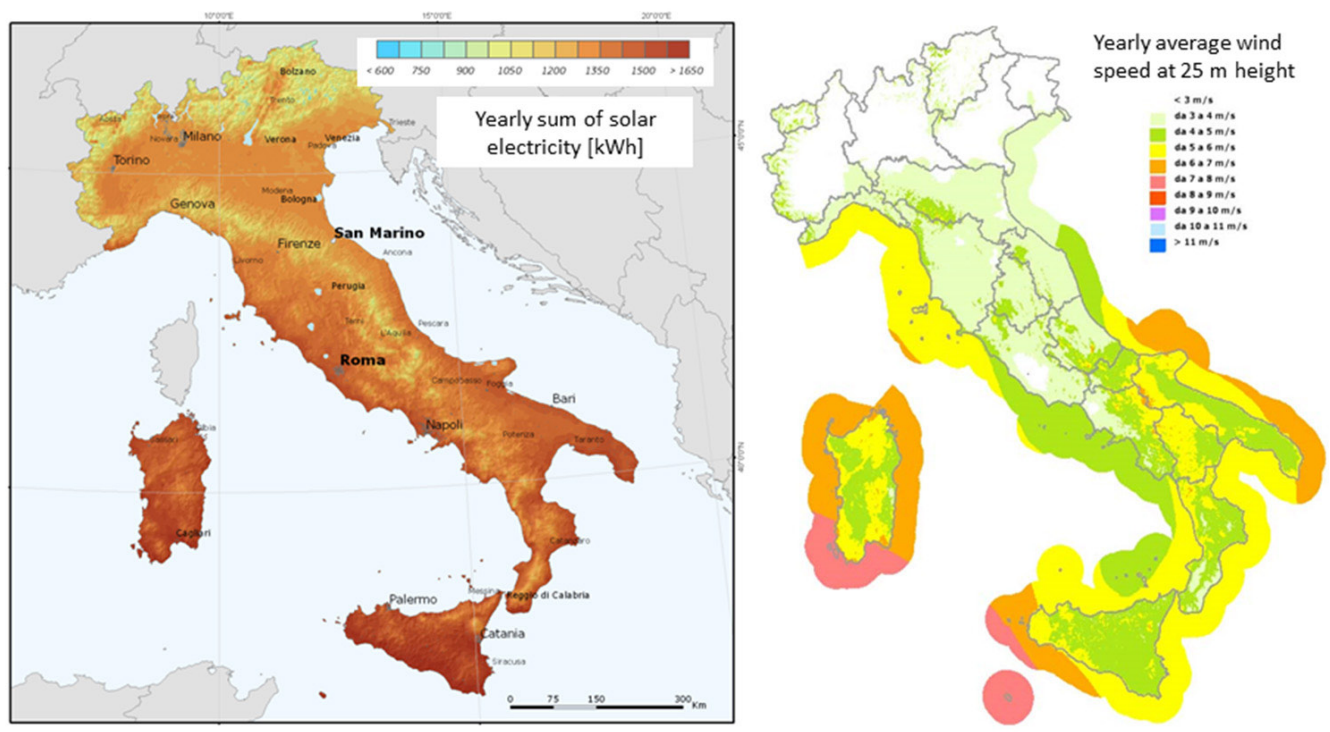

Fig. 8. - Renewable resources usually present a pronounced variability on a geographical basis (source: left PV GIS - JRC [8], right: RSE wind atlas [9]).

Another aspect worth mentioning is the location of renewable resources: in some cases, wind and solar radiation can be effectively exploited in areas far from loads. As example, one can cite the wind farms in Northern Germany and industrial areas in the South, and conversely the wind and solar availability in Southern Italy vs. the Northern loads (fig. 8). Dense urban areas can be more efficient than sparse centres, however they can hardly rely on local wind and solar resources only since (apart from the visual impact) the occupied area is not sufficient to cover the electricity demand. Therefore adequate transmission lines are needed to convey the renewable energy from the generation to the consumption sites. Moreover transmission lines could improve the integration of energy markets for neighbouring countries, allowing to exchange the excess of RES production over a larger geographic area.

\section{3. - Smart grids: definition and technologies}

The widespread diffusion of small-scale generation requires a gradual evolution of the distribution networks from a passive structure to an active one (bidirectional), similar to the transmission network. As mentioned before, this evolution is labelled as "smart grids".

Several elements are involved in this transition, as summarized in fig. 9; in few words a threefold strategy can be implemented:

- smartening the grid ("software" approach, complementary to "hardware" investments on copper and steel); 


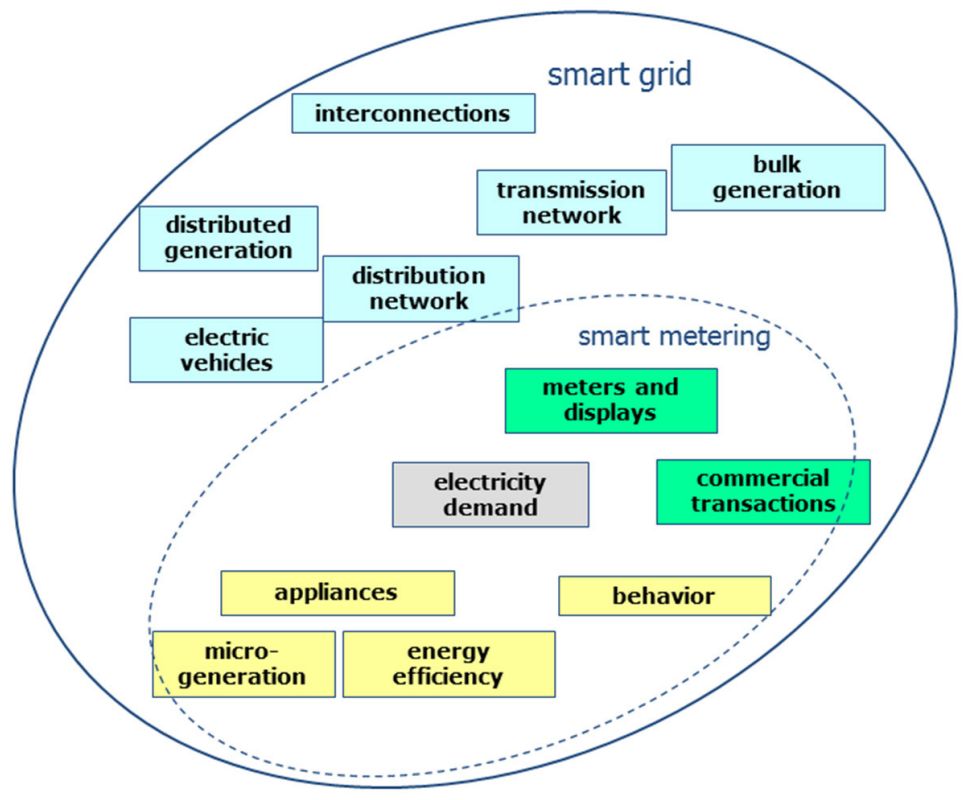

Fig. 9. - Elements of a smart grid (from ERGEG-CEER Position paper on Smart Grids).

- smartening the generators (from simple connection to integration into the system);

- smartening the loads (awareness and participation to the markets).

Regarding the distribution grid, advance monitoring and control techniques include the adoption of protection, measurement and control devices, in order to get a reliable picture of the system's functioning, and to keep currents and voltages within their admissible ranges. In addition, DG's presence requires a stronger coordination between the TSO (in charge of balancing the entire power system, keeping the frequency around $50 \mathrm{~Hz}$ ) and the DSO (in charge of the quality of supply), for guaranteeing the observability of the power system, especially if distributed resources can participate to the ancillary service market. These advanced monitoring and control approaches rely on DSO's assets, such as OLTC, but prospectively on the contribution from distributed resources as well. In liberalized markets, the utilization of storage devices owned and managed directly by network operators is under discussion, since the market rules separated the role of grid operator from the role of generator.

Secondly, generators can be effectively integrated into the system by means of:

- Advanced protection device (to avoid undesired trips).

- Voltage control (exchange reactive power to counterbalance voltage rise caused by injection of active power); this could happen on a local basis, i.e. only on the measurement of parameters at the point of coupling with the network, or following 
set points sent by the DSO. This latter centralized approach could include also modulation of active power for solving congestions, if rules allow DSO to do this.

- Frequency control: automatic reduction of generated power in case of overfrequency (not rewarded if required for system security purposes, usually), or modulation of active power following a signal sent by the TSO (participation on a market basis).

Moving from a system with large bulk generation following the loads, to a framework with the presence of non-programmable generation, entails the need for flexibility from the demand side as well. With Demand Demand Side Integration (DSI) $\left({ }^{5}\right)$, customers change their absorption profile after receiving a price signal or a system signal (security of the system). This allows avoiding over-sizing on the generation side, increasing the efficiency of the generation, reducing the balancing reserve provided by generators, and planning distribution networks on the average load instead of critical peaks. Increased awareness is the first step towards this involvement: a simple in-home-displays can reduce consumption from $3 \%$ to $11 \%$ [10].

"Prosumers" (producer+customers) can get benefits from increased self-consumption, first of all in terms of reduction of the energy bill. Distributed Energy Resources (DER) - including loads, generators and storage devices - can further participate to both local and global flexibility services, also by aggregation, as summarized in table I.

In fig. 10 an example of centralized voltage control for active MV, or LV, networks is given [11]. The control is based on an optimization algorithm that calculates in each time period the set points for the controllable resources with the constraints of maintaining voltages and current within the admissible range. This controller can operate on DSO's owned resources (OLTC, etc.) or third-party equipment (controllable generators/storage units/loads). The algorithm searches for the minimum of the objective function, composed by the sum of "costs" of dispatching the controllable resources. In case there is a regulatory framework allowing DSOs to ask for (remunerated) support, these represent real cost, otherwise the parameters set the priority in the utilization of resources. A typical case where the centralized control is particularly effective is exemplified by the condition of unbalanced MV feeders: one is "active", i.e. the generation is higher than the load, and one "passive", therefore the OLTC cannot set a proper voltage of the MV bus bar to prevent under/over-voltages at the nodes of both feeders. The controller calculates the reactive power modulation from generators and the coordinated operation of OLTC (voltage at MV busbar) in order to solve the issue. It should be observed that voltage controls based on reactive power from generators can increase the losses [12]; the optimization algorithm used in this controller has the additional goal of increasing the efficiency, while satisfying the voltage and current constraints. As a further resource of flexibility for active and reactive power, an electric storage system

$\left({ }^{5}\right)$ DSI refers to all activities focused on advancing the efficiency of electricity utilization, including demand response and energy efficiency. 
TABLE I. - Traditional solutions and "active" solutions for solving various technical issues.

\begin{tabular}{|c|c|c|}
\hline Technical Issue & BAU Distribution Network & $\begin{array}{l}\text { "Smart Grids" } \\
\text { (Active distribution networks) }\end{array}$ \\
\hline Voltage rise/drop & $\begin{array}{l}\text { - Limits/bands for demand and } \\
\text { generation connection/operation } \\
\text { - Generation tripping } \\
\text { - Capacitor banks }\end{array}$ & $\begin{array}{l}\text { - } \text { Coordinated volt-var control } \\
\text { - Static var compensators } \\
\text { - Coordinated dispatch of DER } \\
\text { - On-line reconfiguration }\end{array}$ \\
\hline Hosting Capacity & $\begin{array}{l}\text { - Network reinforcement (e.g., } \\
\text { lines/transformers) }\end{array}$ & $\begin{array}{l}\text { - Coordinated dispatch of DER } \\
\text { - On-line reconfiguration }\end{array}$ \\
\hline $\begin{array}{l}\text { Reactive Power } \\
\text { Support }\end{array}$ & 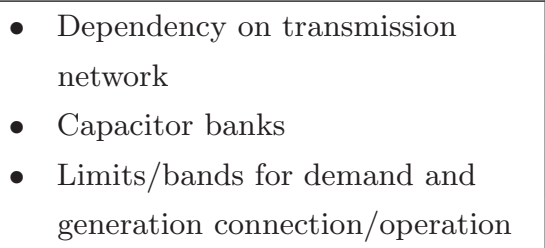 & $\begin{array}{l}\text { - Coordinated volt-var control } \\
\text { - Static var compensators } \\
\text { - Coordinated reactive power } \\
\text { dispatch of DER }\end{array}$ \\
\hline Protection & $\begin{array}{l}\text { - Adjustment of protection settings } \\
\text { - New protection elements } \\
\text { - Limits for generation connection } \\
\text { - Fault ride through specifications } \\
\text { for generation }\end{array}$ & $\begin{array}{l}\text { - On-line reconfiguration } \\
\text { - Dynamic protection settings }\end{array}$ \\
\hline Ageing & $\begin{array}{l}\text { - Strict network designs } \\
\text { specifications based on technical } \\
\text { and economic analyses }\end{array}$ & - Asset condition monitoring \\
\hline
\end{tabular}

(ESS, e.g. a battery) was included; as mentioned earlier, it is under discussion whether DSOs can own ESSs. Testing of this algorithm in real MV networks has demonstrated its effectiveness: depending on the available regulation resources, i.e. OLTC only or also the reactive modulation by dispersed generators, it could increase the Hosting Capacity of more than $20 \%$ with respect to the baseline condition without control [13].

For a comprehensive view of all European projects on smart grid, a survey is published periodically by the Joint Research Centre-JRC [14]: the outlook offers a snapshot of the state of play and of the latest developments in the field of smart grids in Europe. The analysis in the last edition was based on a database of $950 \mathrm{R} \& \mathrm{D}$ and demonstration projects; according to this report, in 2015 a cumulative effort of 5 billion Euros was reached (fig. 11). The document states that "Most of the projects in the database are national projects $(66 \%)$, i.e. projects carried out in one country with the exclusive participation of organisations from that country. Multinational projects, i.e. projects that see the participation of organisations coming from different countries, are less numerous but larger in average investment size (EUR 7.5 million vs. EUR 4.7 million of national projects) [...]. The correlation between a high total number of projects and a 

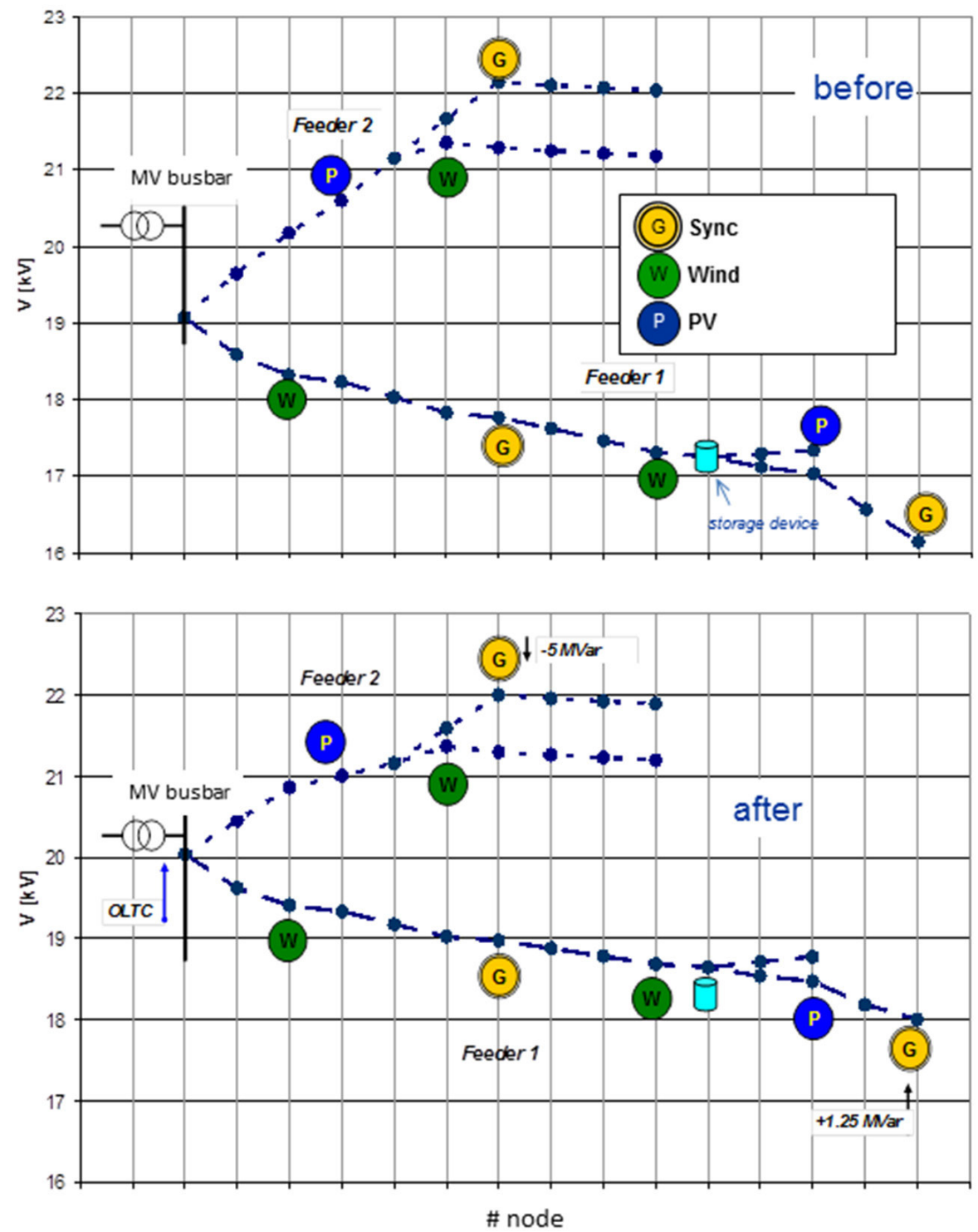

Fig. 10. - Example of centralized voltage control on $20 \mathrm{kV}$ feeders under a primary substation (elaborated from [13]). In case of unbalanced MV feeders (one is "active" and one "passive"), the OLTC cannot solve under/over-voltages (top). The controller requires reactive power modulation from generators and coordinates the operation of OLTC (bottom) (source: RSE).

high number of national projects might be a sign of a mature smart grid environment, where different smart grid stakeholders have had the opportunity to test new technologies and business models thanks to previous $\mathrm{R} \& \mathrm{D}$ and demonstration experiences and to the existence of a supporting national and regulatory framework". 


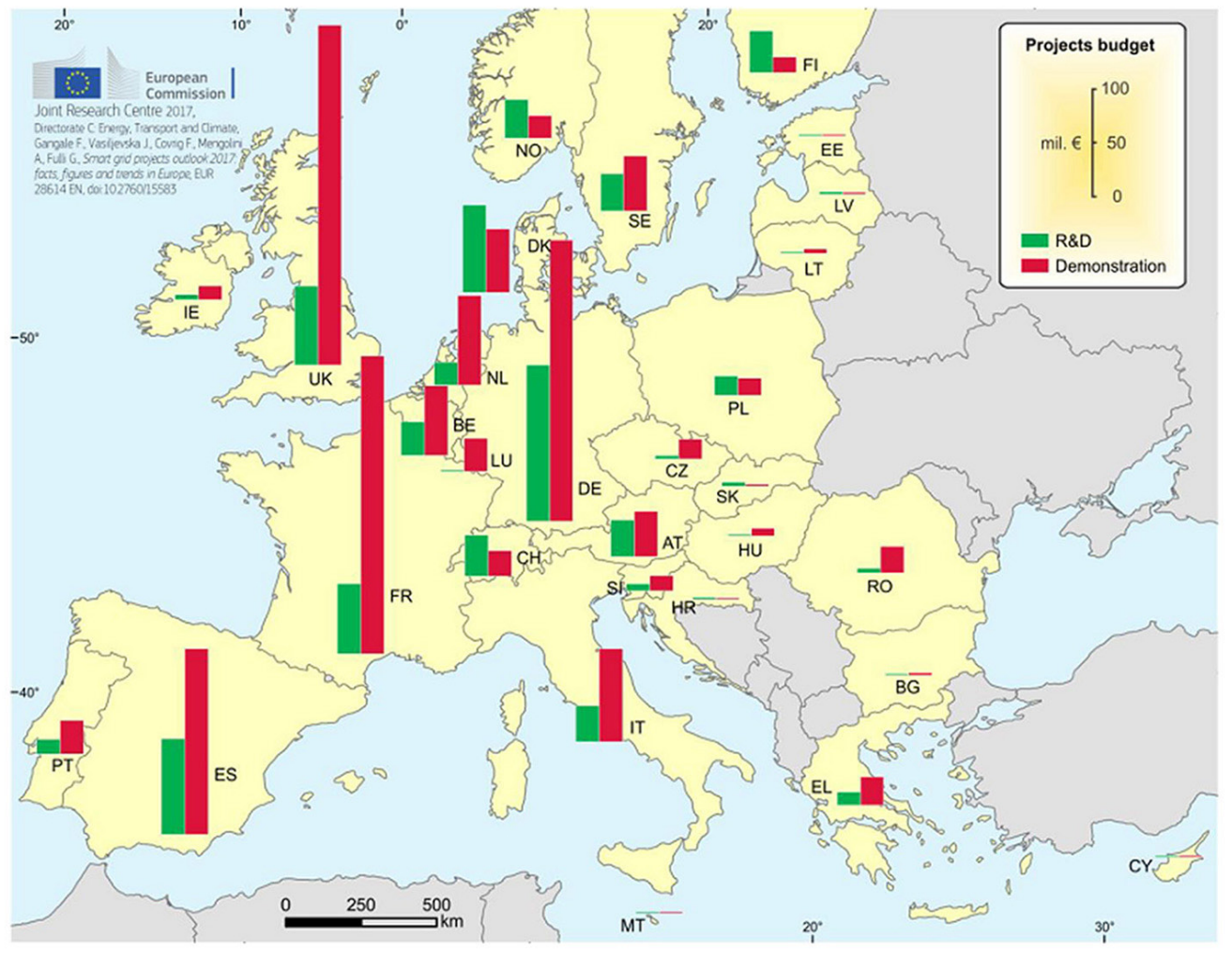

Fig. 11. $-\mathrm{R} \& \mathrm{D}$ and demonstration investments in the EU (source: JRC [14]).

A worldwide initiative on cataloguing smart grid activities was activated by the International Energy Agency Implementing Agreement for a Co-operative Programme on Smart Grids - ISGAN [15].

\section{4. - Estimation of benefits from smart grids}

In a complex scenario, the need of understanding emerges, which technologies among a large number of options are "the best" for solving the given problems. In case of distribution networks, whose investment costs are translated directly to customers' bill, this need is even more acute. In this lecture the focus is limited on the technical benefits, the first step for carrying out the cost-benefit analyses (CBA).

Smart grid solutions are generally evaluated by means of an estimation of the impacts on the performance of the electrical grids. A widely used indicator assesses the quantity of distributed generators that can be connected to a given network without affecting the power quality ("hosting capacity"). Other key performance indicators (KPI) consider the energy efficiency, the voltage stability, and so on; KPIs, and relative calculation methodologies, are now used also by Authorities for rewarding smart grid investments. 

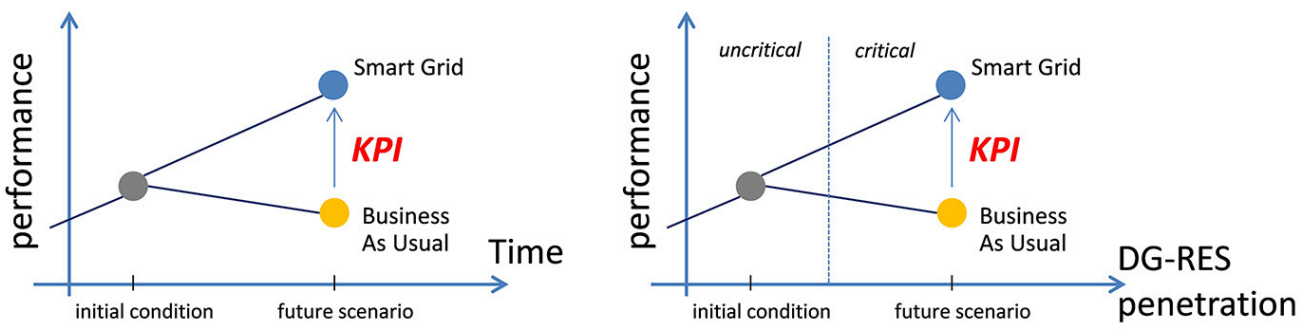

Fig. 12. - Key Performance Indicator calculation schemes: with reference to the time (left) or to the level of RES penetration (right) [16].

With the output based approach, investments are rewarded with reference to the benefit they can bring to the system, not simply on the costs incurred by operators.

KPIs are applied to compare the Business As Usual (BAU) condition with the Smart Grid solution in a given scenario, differing in time or in the level of RES penetration [16]. In this latter approach, the "critical scenario" is characterized by a very high DRES penetration that can reduce the performance of the grid (fig. 12).

The performance evaluation based on data from real networks is likely to be inaccurate due to the fact that there are operation constraints (the presence of real users limits the number and type of tests that can be carried out on the field), as well as the duration and extent of the experimental phase (which would lead to inaccurate statistical indices for the small size of the observed sample). Even in case the real field testing does not pose limitations on the validation of the different parameters, simulations are needed for estimating the alternative conditions not observed in the field test (with vs. without controller).

Given the uncertainty associated with the future scenarios, since the connection of DG is beyond the scope of DSO's assignment and follows other rules for development (availability of primary sources, incentives), a Monte Carlo approach returns the proba-
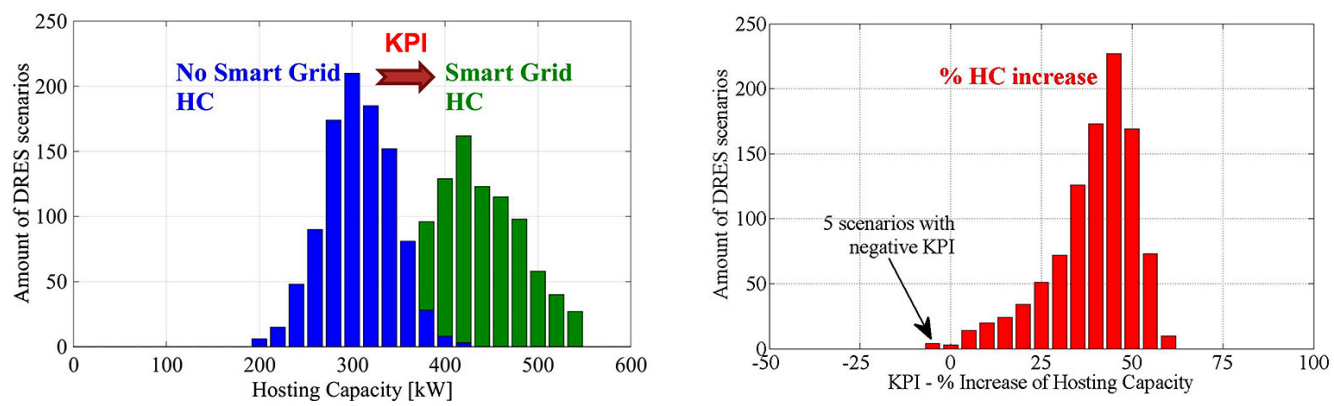

Fig. 13. - Comparison of distribution of Distributed RES scenario as a function of the Hosting Capacity (HC) in the BAU and Smart grid conditions (left); distribution of scenarios as a function of $\mathrm{HC}$ increase with the adoption of the "smart grid" solution (right). Some scenarios show a decrease of $\mathrm{HC}$, i.e. a negative KPI [18]. 
bility distribution of the indicator [17]. For example, the Hosting Capacity is calculated in several scenarios with distinctive size and site of generators' allocation, using realistic profiles for loads and generation. Thanks to this method, it is also possible to conduct sensitivity analysis about allocation of DGs in order to identify combinations of networks and control systems that may, under certain conditions, lead to a decrease of the KPI in the smart grid condition if compared to the BAU situation (fig. 13).

The joint analysis of the different KPIs also allows to extract more information on the different aspects of running active networks such as the inability — in some cases — to increase performance both in terms of hosting capacity and loss containment. This demonstrates how smart grids are enablers for achieving certain goals but they do not represent a "panacea" to solve all issues simultaneously.

\section{5. - Perspectives}

Transitions in the power system last about 50 years, the time necessary to replace generators and upgrade infrastructures. The shift towards renewable resources (RES) observed in these years shows a quicker pace of change than past experiences: smart grids are one of the pillars to enable this transition. Energy systems supplied $100 \%$ RES are probably not reasonable because of several reasons [19], not to mention the availability of sustainable technologies $\left({ }^{6}\right)\left({ }^{7}\right)$, but till 2050 a significant increase is expected both in developing and in OECD countries.

Exploitation of renewable energy resources has led to the diffusion of a large number of small-size generators, connected to distribution grids. Not only farms and factories, but even citizens can now install their own generator, and have "smart" controllers to combine consumption with generation. These small generators could be aggregated to bid their flexibility on the market, competing with conventional large plants for providing ancillary services. This participation, currently limited to few activities, will turn into a necessity within the next decades, when RES penetration will reach higher percentage and fewer thermal plants may remain into service. Integrating variable generation entails additional costs for monitoring and control of distribution networks, and also for balancing the power system, with an increased coordination between the TSO and the DSO.

The progressive reduction of generators' size entails more "lay people" entering into complex interactions with multiple actors (DSO, vendor, aggregator), so shortened and future proof solutions should be defined, also for the administrative procedures. In most countries simplified schemes are in place for rewarding the energy produced by small plants, and more flexible architectures are under discussion (e.g. "energy communities" [20]).

Besides, the electrification of transports and thermal applications may bring a higher RES penetration in the final uses and overall a greater energy efficiency. Liberalization

$\left({ }^{6}\right)$ See the contribution by Vidal to this School.

$\left(^{7}\right)$ See the contribution by Terrasi to this School. 
of retail market allows customers to choose their preferred tariff among a large variety of offers, but this freedom converts into an advantage only if users are aware of their actual consumption habits. Smart meters, delivering consumption data close to real time, support this awareness and allow designing tariff schemes with variable prices, for example depending on the actual RES production in the power system [21].

Buildings are responsible for about $40 \%$ of energy consumption in Europe, and automation is now required in new large buildings together with insulation to reduce the energy wastage [22]. Operators from ICT sector are now proposing appealing applications for energy management: those let customers choose "friendly" solutions, well integrated with other owned devices (smartphones primarily). Smart appliances are allowing people to remotely programme their utilization easily, also by means of complex but plain energy management systems. On the other hand, such solutions are often based on proprietary protocols for collecting and managing data in remote cloud servers; apart from privacy and security issues, this may lead to a lack of interoperability among solutions (customers cannot easily integrate devices from different manufacturers), and in the worst case a loss of functionalities when the internet connection is absent, or the manufacturer discontinues the software support.

Basically, smart grids are composed of the superimposition of the power grid and the ICT layer: development of successful and cost effective solutions needs the close collaboration of experts from both sectors, and even the support of social sciences to actually enable customers' participation apart from pure economic reward. Last but not least, regulation authorities have the duty of proposing shared methodologies to assess benefits and costs of smart grid development, and fairly allocate these costs to the different actors. In any case, "smart technologies" are the way to develop a sustainable energy system, not the goal.

\section{REFERENCES}

[1] National Renewable Energy Action Plan (NREP), February 2011 http://www.eea. europa.eu/highlights/renewable-energy-production-must-grow.

[2] Ohler C., "The physics of power systems operation" in Lectures Notes - Joint EPS-SIF International School on Energy, Course 2, Energy: Basic Concepts and Forefront Ideas, EPJ Web of Conferences, 98 (2015) 03002.

[3] CENELEC, technical standard EN 50160, "Voltage Characteristics of electricity supplied by Public Distribution Systems", 2010.

[4] Autorità di Regolazione per Energia Reti Ambiente (ARERA, former AEEGSI), "Relazione annuale 2016", 2017 (in Italian), www.arera.it.

[5] Delfanti M., Pasquadibisceglie M. S., Pozzi M., Gallanti M. and Vailati R., "Limits to Distributed generation on Italian MV networks. Electricity Distribution - Part 1", in Proceedings of the 20th International Conference and Exhibition; 8-11 June 2009 (CIRED) 2009, pp. 1-4.

[6] Kotsampopoulos P. et al., "EMC Issues in the Interaction Between Smart Meters and Power-Electronic Interfaces", IEEE Trans. Power Delivery, 32 (2017) 822.

[7] Union for the Co-ordination of Transmission of Electricity UCTE, "Final Report - System Disturbance on 4 November 2006", www.entsoe.eu. 
[8] Huld T., Šúri M., Dunlop E., Albuisson M. and Wald L., "Integration of HelioClim-1 database into PVGIS to estimate solar electricity potential in Africa", in Proceedings of the 20th European Photovoltaic Solar Energy Conference and Exhibition, 6-10 June 2005, Barcelona, Spain, 2005 http://re.jrc.ec.europa.eu/pvgis/.

[9] Ricerca sul Sistema Energetico - RSE SpA, ATLAEOLICO, http://atlanteeolico. rse-web.it/.

[10] Darby S., "Communicating energy demand: measurement, display and the language of things", in Engaging the Public with Climate Change: Behaviour Change and Communication, edited by Whitmarsh L., O'Neill S. and Lorenzoni I. (Earthscan) 2010.

[11] Bosisio A., Moneta D., Vespucci M. T. and Zigrino S., "A procedure for the optimal management of medium-voltage $\mathrm{AC}$ networks with distributed generation and storage devices", AIRO Proceedings, Procedia Soc. Behav. Sci., 108 (2014) 164.

[12] Sauer P. W., "Reactive Power and Voltage Control issues in Electric Power Systems", in Applied Mathematics for Restructured Electric Power Systems, edited by CHOw J. H. and Wu F. F. (Springer, Boston) 2005, pp. 11-24.

[13] Stein D., Consiglio L., Viganò G., Michelangeli C., Carlini C. and Moneta D., "Real field testing results of the innovative Medium Voltage control system developed in the Italian Demonstrator of GRID4EU", Proceedings 46th Session CIGRE, Paris, 2016, www. cigre.org.

[14] Gangale F., Vasiljevska J., Covrig F., Mengolini A. and Fulli G., "Smart grid projects outlook 2017: facts, figures and trends in Europe", (Publication Office of the European Union) 2017.

[15] ISGAN, "Global Smart Grid Inventory" http://www.iea-isgan.org/bbs/content.php? co_id=sub2_2a.

[16] IGreenGRID Europeana Project, "KPIs calculation procedure and results", http://www . igreengrid-fp7.eu/.

[17] Rossi M., Viganò and Moneta D., "Hosting capacity of distribution networks: Evaluation of the network congestion risk due to distributed generation", in Proceedings of the 2015 International Conference on Clean Electrical Power (IEEE ICCEP) 2015.

[18] Jarela J., Hatziargyriou N., Puglisi L., Rossi M., Abart A. and Bletterie B., "The IGREENGrid Project: Increasing Hosting Capacity in Distribution Grids", IEEE Power Energy Mag., 15 (2017) 30.

[19] Wagner F., "Electricity generation by intermittent sources", in Lectures Notes - Joint EPS-SIF International School on Energy, Course 2, Energy: Basic Concepts and Forefront Ideas, EPJ Web of Conferences, 98 (2015) 04006.

[20] European Commission, Communication to the European Parliament, the Council, the European Economic and Social Committee, the Committee of the Regions and the European Investment Bank, "Clean Energy For All Europeans" (Winter package'), $\operatorname{COM}(2016) 860$ final, Brussels, 2016.

[21] Moneta D. and Mauri G., "Factors Influencing Adoption of Smart Meters", in Smart Grid Handbook, edited by Liu C. C., McArthur S. and LeE S. J. (John Wiley and Sons Ltd, Chichester, UK) 2016, pp. 1119-1131.

[22] Directive 2012/27/EU of the European Parliament and of the Council of 25 October 2012 on energy efficiency, amending Directives 2009/125/EC and 2010/30/EU and repealing Directives 2004/8/EC and 2006/32/EC. 